\title{
NIVELES DE CORTISOL SALIVAL Y TIPOS DE PERSONALIDAD DE GROSSARTH-MATICEK Y EYSENCK: UN ESTUDIO TRANSCULTURAL
}

\author{
SANTOS OREJUdO $^{1}$, Everardo CAMACHO ${ }^{2}$ Y ClaUdia VEGA-MicheL $^{2}$ \\ ${ }^{1}$ Departamento de Psicología y Sociología, Universidad de Zaragoza, Zaragoza, España \\ ${ }^{2}$ Instituto Tecnológico de Estudios Superiores de Occidente-ITESO, Tlaquepaque, Jalisco, México
}

\begin{abstract}
Resumen: Basándonos en la tipología de personalidad de Grossarth-Maticek y Eysenck que relaciona ciertos tipos de personalidad con el padecimiento de enfermedades, en esta investigación se analiza en dos muestras de estudiantes universitarios mexicanos y españoles la relación entre el tipo de personalidad de propensión al cáncer y el de propensión a las enfermedades cardiovasculares y el consumo de tabaco y los niveles de cortisol salival. Un total de 190 estudiantes mexicanos y españoles respondieron al cuestionario de personalidad Short Interpersonal Reactions Inventory (SIRI) y se les tomaron muestras de saliva antes y después de esta tarea. Los resultados ponen de manifiesto que el efecto más importante es el relativo al país, con niveles de cortisol más altos para los estudiantes mexicanos. Por otra parte, únicamente en el caso de éstos aparece que el tabaco y la personalidad aumentan los niveles de cortisol. Se discuten los resultados desde la teoría de Grossarth-Maticek y Eysenck y también desde la perspectiva de las diferencias entre países y del cortisol como riesgo para la salud.
\end{abstract}

Palabras clave: Cortisol; tipos de personalidad; tipos de reacción al estrés; tabaco; estrés; estudios transculturales.

Levels of salivary cortisol and personality types of Grossarth-Maticek and Eysenck: A cross-cultural study

\begin{abstract}
Based on Grossarth-Maticek and Eysenck's personality typology which relates types of personality with certain diseases, this study analyzes, in two samples of Mexican and Spanish students, the relationship between personality type and the propensity for cancer and the propensity for cardiovascular disease, and tobacco consumption and the levels of salivary cortisol. A total of 190 Mexican and Spanish university students responded to the Short Interpersonal Reactions Inventory (SIRI) while we took saliva samples before and after the task. The results reveal that the more important effect came from the country of origin, with higher cortisol levels in the Mexican students. On the other hand, only in these students tobacco use and the type of personality were found to increase cortisol levels. The results are discussed from the perspective of Grossarth-Maticek and Eysenck's theory as well as from the perspective of differences between countries and cortisol as a health risk.
\end{abstract}

Keywords: Cortisol; personality types; stress behavior types; tobacco; stress; cross-cultural study.

Actualmente son bien conocidos los trabajos de Grossarth-Maticek y Eysenck sobre las relaciones entre su tipología de personalidad (tipos de reacción al estrés) y la salud. Sobre tres estudios iniciales (Eysenck, 1987a,b, 1991a;

Recibido: 3 abril 2012; aceptado: 5 mayo 2012.

Correspondencia: Santos Orejudo Hernández, San Juan Bosco 7, 50009 Zaragoza, España.

Correo-e: sorejudo@unizar.es
Eysenck, Grossarth-Maticek y Everitt, 1991; Grossarth-Maticek, Eyesenck y Vetter, 1988), planteaban una tipología de personalidad definida según el modo de reaccionar al estrés, que predecía el cáncer, las enfermedades coronarias y el permanecer sano. Teóricamente se asumía que los factores de riesgo tradicionales interactuaban con la personalidad para potenciar sus efectos negativos sobre la salud (Eysenck, 1988, 1991b; Eysenck et al., 1991; Grossarth-Maticek 
et al., 1988). La actuación de tales factores supondría una mediación del sistema inmunológico y del sistema endocrino y la interacción entre ellos (Eysenck, 1991c). También se presentaba un modelo de intervención sobre la personalidad basado en los principios de la terapia de conducta que permitía modificar las características patológicas de las mismas y reducir los riesgos de mortalidad en aquellas personas que presentaban las personalidades de predisposición a la enfermedad (GrossarthMaticek y Eysenck, 1991; Eysenck y GrossarthMaticek, 1991). Revisiones amplias se pueden encontrar en Eysenck (1991a), Sandín (1995), Orejudo, Froján y Malo (2004), y Sebastián y Miret (2009).

Tras la aparición de estos primeros trabajos y por la espectacularidad de sus resultados, se publicaron distintos estudios comentando aspectos críticos sobre el procedimiento de recogida de los datos o con algunas incosistencias encontradas en los mismos (Amelang, 1991; Kiecolt-Glaser y Chee, 1991; Pelossy y Apleby, 1992). Fruto de estas críticas, surgieron nuevos seguimientos de las muestras confirmando los resultados previamente encontrados (Eysenck, 1991d, 1993). Los autores también presentaron nuevas versiones de los instrumentos que sobre el mismo soporte teórico presentaban otros instrumentos de medida, tales como las escalas de autorregulación y diferenciación (GrossarhtMaticek y Eysenck, 1995; Kirk y Martín, 1998).

Posteriormente se han venido publicando trabajos de grupos independientes en distintos países que han adaptado las escalas a sus respectivas lenguas. En general, se ha encontrado que hay una clara distinción entre los tipos salutógenos y los de predisposición coronaria, y que los tipos de personalidad guardan una estrecha relación con otros constructos como el neuroticismo, la extroversión, la depresión, los estilos de afrontamiento, la competencia personal, la alexitimia, la hostilidad, el apoyo social, el opitmismo o con variables sociodemográficas y con estilos de vida (Amelang y Schmitz-Rathjens, 1992, 1996; Consoli, Cordier y Ducimetiere, 1993; Flebus y Cherri, 2003; Martínez y Reyes, 2004, 2007; Orejudo y Froján, 2004; Sandín, Chorot, Jiménez y Santed, 1994; Sandín, Chorot, Navas y Santed, 1992; Sandín,
Chorot, Santed y Jiménez, 1994). En cuanto al poder predictivo, se comprueba que los tipos de personalidad se asocian a la presencia de síntomas y a conductas relacionadas con la salud como la nutrición, el ejercicio físico, la automedicación o la frecuencia de visitas al médico (Larsson, Nordström, Ljunggren y Nyberg, 1995; Orejudo, Froján y Malo, 2007), pero otros estudios no confirman estos resultados (Rodríguez, Lemos y Canga, 2001, 2002). También se han encontrado algunas limitaciones, tales como la no diferenciación de los tipos de predisposición cardiovascular y del cáncer, la escasa relevancia de esta tipología al compararlas con otros constructos del campo de la salud (Amelang y Schmidt-Rathjens, 1992; Amelang, 1997; Orejudo y Froján, 2005), o problemas con el sistema de categorización de los sujetos en función de sus puntuaciones en las distintas escalas (Larson, et al, 1995).

Un último aspecto tiene que ver con los trabajos que han analizado las relaciones con indicadores directos de enfermedad. Aquí, las metodologías han sido diversas, desde estudios de casos y controles, estudios retrospectivos y algunos estudios longitudinales. Así, QuanderBlaznik (1991), en una muestra de 76 personas que acuden a una biopsia, encuentra que el tipo 1 , junto con la edad y el número de cigarrillos, discrimina entre los sujetos que sufren cáncer de pulmón de aquellos que no lo tienen. En estudios retrospectivos, en algunos casos se encuentran algunas asociaciones entre los tipos de personalidad y enfermedades como el cáncer, las alergias, la úlcera, la hipertensión o el infarto (Schmitz, 1992), pero en otros los resultados son mucho más pobres (Smedslung, 1995; Terada, Kawakami, Inaba, Takatsuka y Shimizu, 2000). Con otras enfermedades, se ha encontrado que los componentes del tipo 1 de personalidad pueden discriminar la gravedad de la enfermedad en pacientes con hepatitis C (Nagano, Nagase, Sudo, y Kubo, 2004), pero estas mismas variables no predicen qué mujeres que acuden a una biopsia acabarán teniendo un cáncer de mama (Sebastián y Miret, 2009). Por su parte, Matthews, Yousfi, Schmidt-Rathjens y Amelang (2003) encuentran en un estudio transversal que una combinación de puntuaciones factoriales, en la que tanto el tipo de predispo- 
sición al cáncer como el tipo de predisposición a la enfermedad cardiovascular tiene una alta saturación, discrimina entre grupos de personas que autoinforman múltiples enfermedades y enfermedades coronarias de aquellos que permanecen sanos. Debemos añadir otros dos estudios prospectivos con resultados contradictorios. Así, en una nueva publicación de GrossarthMaticek et al. (2000) en la que, con muestras mucho mayores que las de sus estudios originales, una combinación de las puntuaciones de los tipos de personalidad de predisposición a la enfermedad, la corrección dinámica del cuestionario, predice conjuntamente con otros factores de riesgo la aparición del cáncer de mama de manera prospectiva. Por otra parte, también se conocen los resultados de un estudio longitudinal japonés con una muestra de 27.000 personas seguidas durante 7 años y usando únicamente una escala similar al tipo 1 de Grossarth-Maticek y Eysenck, la escala de racionalidad /antiemocionalidad (Hirokawa, Nagata, Takatsuka y Shimizu, 2004). Los resultados de este estudio ponen de manifiesto una ligera relación de este tipo de personalidad con el cáncer o las enfermedades cardiovasculares, pero quedan muy limitadas cuando se controlan otras variables como la edad, por lo que en opinión de los autores, no se puede decir que la racionalidad y la antiemocionalidad se relacionen con estas patologías, señalando que probablemente las diferencias culturales pueden ser muy importantes, por cuanto en Japón estas características tienen un alto valor adaptativo.

Otros constructos de personalidad se han usado en la literatura para tratar de dar cuenta de los distintos niveles de cortisol en plasma encontrados así como de otros trastornos y enfermedades (Álvaro y Traver, 2010; Hauner et al, 2008; Fernández del Rio, López y Becoña, 2011; Orejudo y Froján, 2005). El supuesto de partida en muchos de estos casos es que se da una interacción entre la personalidad y el estrés. Así, existen bastantes estudios que, basándose en los tipos de neuroticismo y extraversión/introversión de Eysenck, relacionan las variables de personalidad y los niveles de cortisol como indicadores de estrés. Por ejemplo, Hauner et al. (2008) evaluaron en 230 adolescentes las dimensiones de neuroticismo y extraversión/ introversión, encontrando que los patrones circadianos de cortisol son más planos en el caso de los hombres con altas puntuaciones en neuroticismo. Por su parte, los participantes con altos niveles de introversión mostraban mayores niveles de cortisol. Otras medidas de esta hormona implican la aplicación de dexametasona (que suprime los niveles de cortisol) con objeto de evaluar de forma dinámica la autoregulación del eje hipotálamo-hipófiso-corticosuprarrenal (HHC) para regresar a niveles basales. Zobel et al. (2004) encontraron en 92 sujetos sanos que altos niveles de neuroticismo están asociados con la desregulación del eje HHC. Este indicador se vuelve predictor de riesgo y vulnerabilidad para la depresión mayor. García de la Banda et al. (2004) evaluaron los niveles de cortisol bajo condiciones de estrés (hablar en público) encontrando que los sujetos altos en neuroticismo y extraversión tenían mayores niveles de cortisol al realizar la tarea estresante. Datos semejantes son informados por Portella, Harmer, Flint, Cowen y Goodwin (2005). En semejanza a la dimensión de introversión, se han realizado estudios en niños con rasgos de nula emocionalidad encontrando altas correlaciones con niveles bajos de cortisol (Loney, Butler, Lima, Counts y Eckel, 2006). Vermunt, Peeters y Berggren (2007) refieren correlaciones positivas entre cortisol salival y puntuaciones altas en personalidad tipo A (hiperactividad, irritabilidad, agresividad, competitividad, impaciencia e impulsividad).

Otras clasificaciones usan las categorías de personalidad resiliente (flexible en control del ego y alto en capacidad de adaptación al medio cambiante), personalidad controlada (baja resiliencia y alto en control del ego), y personalidad incontrolada (bajo en las dos dimensiones). Hart, Burock, London, Atkins y Bonilla-Santiago (2005), evaluaron a 63 niños a través de sus comportamientos observados en el aula y al clasificarlos encontraron que la personalidad resiliente era la que se asociaba a mayores niveles de cortisol en general, si bien los otros dos tipos se asociaban a mayores cambios (reactividad) bajo condiciones de estrés en los niveles de cortisol. De este modo se considera que el neuroticismo es un mediador importante en la relación estrés-enfermedad, en la que la disre- 
gulación del eje HHC es un indicador crónico del proceso de enfermarse (Ranchor y Sanderman, 1991).

Aunque algunos autores como Oswald et al. (2006) señalan que existe evidencia débil de la relación entre el estrés y la personalidad, y que este tipo de relación requiere clarificación, otros autores sugieren modificaciones metodológicas como exponer de forma repetida a la fuente de estrés a los sujetos bajo estudio, analizar el área bajo la curva de cortisol y la agregación de datos para evidenciar con mayor claridad el grado de vínculo entre estos dos factores (Pruessner et al., 1997). Estos autores encuentran que la imagen de una persona insegura, controlada externamente y «subdominante» tiene mayores niveles de cortisol salival.

Aunque hasta el presente se han aportado numerosos datos sobre la tipología de Grossarth-Maticek y Eyensenck, apenas hay investigaciones que hayan analizado la hipótesis de la mediación del cortisol (Eysenck, 1991c). Únicamente en el caso de Larson et al. (1995), en el que se analizan marcadores biológicos, se encontraron algunas diferencias entre grupos de sujetos con personalidades saludables y no saludables en los niveles de colesterol, triglicéridos y el volumen corpuscular medio, pero en ningún otro marcador de tipo inmunitario. Por ello, entendemos que son necesarios nuevos estudios que intenten abordar de una manera novedosa las relaciones entre los tipos de personalidad y algún marcador biológico de tipo inmunitario o endocrino. Por ello, el objetivo de este trabajo es analizar las relaciones entre las tipologías de personalidad de GrossarthMaticek y Eysenck y los niveles de cortisol salival en estudiantes universitarios de dos países distintos, España y México. Se hipotetiza que las puntuaciones obtenidas en las escalas de propensión a la enfermedad de GrosarthMaticek y Eysenck correlacionarán con los niveles de cortisol salival, de tal manera que los sujetos que presenten altas puntuaciones en las escala Tipo 1 (propensión al cáncer) presentarán mayores niveles de cortisol. Este efecto podría aparecer igualmente en el tipo 2 (predisposición a la enfermedad coronaria), en el que se encuentran algunas diferencias en el afrontamiento del estrés (Orejudo y Froján, 2004), pero para el que no se establece que el cortisol esté mediando en la aparición de la enfermedad. Se presupone además que se dará una interacción con otros factores de riesgo clásicos como el consumo de tabaco, encontrándose en estos casos mayores niveles de cortisol cuando coexistan con altas puntuaciones en las tipologías de personalidad Este resultado, a priori, debería ser independiente de la nacionalidad de los participantes.

\section{MÉTODO}

\section{Participantes}

Se han recogido los datos de un total de 190 estudiantes universitarios mexicanos (88, $46,3 \%$ ) y españoles $(102,53,7 \%)$. El $69 \%$ son mujeres mientras que un $31 \%$ son varones, siendo el porcentaje de chicas ligeramente superior en el grupo de estudiantes españoles, pero sin que las diferencias sean estadísticamente significativas $\left(\chi^{2}=1,18\right)$. La edad media del grupo es de 21,3 $(D T=4,4)$ años, sin que las diferencias entre los mexicanos $(20,82)$ y los españoles $(21,77)$ sea estadísticamente significativa $\left(\mathrm{F}_{(1,183)}=2,2\right)$. El rango de edad oscila entre los 17 y los 30 años entre los estudiantes mexicanos y entre los 17 y los 52 en el caso de los españoles. Por titulación, los estudiantes mexicanos cursan estudios de Psicología $(80,5 \%)$, Relaciones Industriales $(18,4 \%)$ y Contaduría (1,1\%), en el Instituto Tecnológico de Estudios Superiores de Occidente, en el estado de Jalisco. En el caso de los estudiantes españoles eran estudiantes de la Facultad de Educación de Zaragoza, de Magisterio de Educación Musical (36,2\%), de Audición y Lenguaje $(32.3 \%)$ y de Psicopedagogía $(31,5 \%)$

Todos ellos fueron reclutados al aceptar la invitación de los organizadores de la investigación a participar de manera voluntaria en la misma. El procedimiento de muestreo fue no aleatorio, por disponibilidad si se encontraban en las aulas en el momento en el que los investigadores realizaron la toma de datos. La única condición para participar en la investigación era hacerlo voluntariamente y no padecer ninguna enfermedad grave. El procedimiento de toma 
de las muestras de saliva para analizar los niveles de cortisol se hizo por la tarde, y el tiempo entre ambas tomas no excedió los 30 minutos.

\section{Procedimientos de evaluación}

Short Interpersonal Reactions Inventory (SIRI; Grossarth-Maticek y Eysenck, 1990). Se utilizó la versión española adaptada por Sandín et al. (1992). Consta de 70 items que evalúan los seis tipos de reacción al estrés, i.e., tipo 1 (predisposición al cáncer), tipo 2 (predisposición a la enfermedad coronaria), tipo 3 (histérico), tipo 4 (saludable; incluye dos formas), tipo 5 (racional-antiemocional), y tipo 6 (antisocial). Los participantes contestan a cada uno de los items indicando si lo que indica es para él verdadero (0) o falso (1). En esta investigación se han usado únicamente los tipos 1 $(\alpha=0,71)$ y $2(\alpha=0,60)$. Las puntuaciones de ambas escalas se obtienen sumando las respuestas afirmativas a cada uno de los 10 ítems de la escala que señala características típicas de cada personalidad. Aparte de estas puntuaciones cuantitativas, se han considerado dos grupos de sujetos en cada caso en función de sus puntuaciones en cada escala, i.e., aquellos que tienen puntuaciones de 0 o 4 (baja puntuación en el tipo) y los que tienen entre 5 y 10 (alta puntuación en el tipo).

Autoinforme de estilos de vida. Con el objetivo de controlar posibles variables extrañas relacionadas con el patrón de sueño o con otros estilos de vida, mediante un autoinforme se preguntaba a los participantes por otras conductas como el consumo de alcohol, tabaco, el número de horas que habían dormido o la hora de despertar. Igualmente se consultó sobre el grado de salud (tener o no tener alguna enfermedad) y seguir o no un tratamiento farmacológico.

Evaluación del cortisol salival. La medida de la concentración de cortisol en saliva se analizó mediante la técnica de inmunoensayo ELISA (enzime-linked immunoabsorbent assay; basado en la competición entre el antígeno no unido y el antígeno unido a enzima), determinándose la concentración libre de cortisol salival expresada en nanomoles por litro (nmol/L).
El análisis de cortisol salival es un procedimiento no invasivo que tiene una alta correlación con los niveles de cortisol en sangre, de hasta el 98\% (Kirschbaum y Hellhammer, 1989). Las medidas de cortisol varían con relación a la norma en función de distintas condiciones como la hora del día, mayor variabilidad en las horas de la mañana, o variables personales relacionadas con el patrón de sueño (Kudielka, Federanko, Hellhammer y Wüst, 2006). Igualmente, tal como se hipotetiza en este trabajo, los niveles de cortisol pueden variar en función de los niveles de estrés o de distintos procesos de enfermedad como el cáncer o las enfermedades autoinmunes (Kiecolt-Glasser, McGuire, Robles y Glaser (2002), o por efectos directos de determinados tratamientos farmacológicos como los asociados a los tratamientos anticonceptivos con hormonas. Asimismo influyen factores como la edad, el género, el embarazo, la lactancia, el fumar, el consumo de alcohol o suplementos dietéticos (Kudielka, Hellhammer y Wüst, 2009). Para controlar estas variables se incluyó un autoinforme en el que se preguntaba a los participantes si padecían algún tipo de enfermedad y si seguían algún tipo de tratamiento. Como ya hemos comentado, se tomaron dos medidas de cortisol, una antes de rellenar las pruebas de personalidad y otra después, recogiéndose todos los datos en horario de tarde, para evitar posibles efectos asociados al ciclo circadiano de la hormona.

\section{Procedimiento}

En el caso de los estudiantes mexicanos se les citaba por la tarde a un salón con 25 ordenadores en los que se aplicaban las pruebas de personalidad. Antes de empezar se les pedía que se enjuagasen la boca y recogiesen un mililitro de saliva en un tubo de polipropileno. La misma petición era realizada una vez acaba la tarea de recogida de datos. En la investigación, aparte de los instrumentos de evaluación indicados, rellenaron otros cuestionarios por lo que entre la primera y la segunda recogida de muestras de saliva podían pasar entre 25 y 30 minutos. A los estudiantes se les garantizaba que el uso de los datos era para investigación y que lo que 
realmente importaba era valor del grupo, pero que si alguno de ellos tenía interés, sería informado de sus resultados de manera individual.

Por lo que respecta a los españoles, el procedimiento era el mismo, con la única diferencia de que los cuestionarios fueron rellenados en formato de lápiz y papel y el aula en la que se hizo la toma de datos era la misma en la que habitualmente participaban en las clases. Bajo estas condiciones, ni los estudiantes españoles ni los mexicanos se encontraban ante un estresor al rellenar los cuestionarios o tomar las muestras de saliva.

Todas las muestras de cortisol fueron analizadas en el Laboratorio de Psicofisiología del Instituto Tecnológico de Estudios Superiores de Occidente (México), por lo que las muestras de los estudiantes españoles fueron trasladadas desde España. Los niveles de cortisol en saliva a temperatura ambiente no se ven afectados los primeros 3 meses o el primer 1 año con las muestras congeladas (Hansen, Garde y Persson, 2008). En algunos casos no se dispone de los datos de cortisol de algunos participantes, concretamente 15 de la primera toma y otros 15 de la segunda., ya que para el análisis se necesitaba por lo menos $1 \mathrm{~mL}$ de muestra de saliva y no se cumplió este requisito.

\section{RESULTADOS}

Por países, encontramos alguna diferencia en los estilos de vida. Así, el porcentaje de fumadores entre los estudiantes mexicanos es significativamente mayor que entre los españoles, $51,5 \%$ entre los mexicanos y $36,6 \%$ entre los españoles $\left(\chi^{2}=4,4, p<0,05\right)$ En cuanto a las puntuaciones en las dos escalas de personalidad consideradas (Tabla 1), no hay diferencias significativas y en ambos casos se puntúa de manera parecida $\left(F_{(1,153)}=0,05 \mathrm{y} \mathrm{F}_{(1,154)}=0,017\right)$. De manera global, se encuentra una correlación significativa entre las puntuaciones del tipo $1 \mathrm{y}$ los niveles de cortisol encontrados en la primera medida $(r=0,20, p<0,01, n=155)$. Al analizar específicamente lo que ocurre en cada país, podemos ver cómo esta relación únicamente se da entre los estudiantes mexicanos $(r$ $=0,28, p<0,01)$.

Por otra parte, se encuentra una reducción del nivel de cortisol entre los dos momentos de medida del mismo $\left(t_{(159)}=2,1, p<0,05\right)$, de tal manera que la primera medida presenta mayores niveles de cortisol que la segunda $[19,45$ $(17,19)$ vs. $17,53(14,52)]$. Las puntuaciones obtenidas en ambos momentos muestran una alta correlación entre sí $(r=0,75, p<0,001)$. $\mathrm{Al}$ analizar los cambios entre las dos medidas de cortisol con análisis de varianza de medidas repetidas en un factor, se encuentra que el tiempo que transcurre entre los dos momentos favorece una reducción del nivel general de cortisol $\left(F_{(1,158)}=5,2 ; p<0,05, \eta_{p}^{2}=0,032\right)$, pero que al dividir al grupo en función de los niveles previos de cortisol, aparece un nuevo efecto que indica que el cambio en los niveles de cortisol entre el primer y el segundo momento dependen de los niveles previos de cortisol $\left(F_{(1,158)}=24,6\right.$; $\left.p<0,001, \eta_{p}^{2}=0,135\right)$. Así, el grupo de sujetos que presentaban el $50 \%$ de niveles de cortisol más bajos, suben ligeramente sus niveles $[7,023$ $(5,95)$ vs. 9,316 $(8,51)]$, mientras que por el contrario, aquellos que mostraban los niveles más elevados tienden a reducirlos $[31,92$ $(15,67)$ a $25,75(14,7)]$.

Tabla 1. Valores medios de cortisol salival (nmol/L) en los dos momentos de medida en ambas muestras (española y mexicana) y de las puntuaciones en los tipos 1 y 2 de personalidad.

\begin{tabular}{|c|c|c|c|c|c|c|}
\hline & \multicolumn{3}{|c|}{ España } & \multicolumn{3}{|c|}{ México } \\
\hline & $n$ & Media & $D T$ & $n$ & Media & $D T$ \\
\hline Tipo 1 & 81 & 2,48 & 2,08 & 88 & 2,64 & 2,16 \\
\hline Tipo 2 & 82 & 2,73 & 1,92 & 88 & 2,70 & 2,31 \\
\hline Cortisol-1 & 88 & 11,58 & 11,38 & 87 & 26,62 & 17,98 \\
\hline Cortisol-2 & 74 & 11,66 & 12,55 & 87 & 23,27 & 12,92 \\
\hline Cortisol-1 menos Cortisol-2 & 74 & 0,32 & 12,35 & 86 & 3,32 & 10,70 \\
\hline
\end{tabular}


Para comprobar el efecto de las variables consideradas en la investigación sobre los niveles de cortisol se han llevado a cabo dos análisis de varianza multivariados (MANOVA), tomando como variables dependientes las dos medidas de cortisol, y como independientes el país, el consumo de tabaco diferenciando grupos de fumadores y no fumadores y las puntuaciones en los tipos de personalidad estableciendo los agrupamientos ya señalados (sujetos altos vs. bajos en el tipo de personalidad). En el primer análisis se incluyó el tipo 1 de personalidad, y en el segundo análisis se incluyó el tipo 2 de personalidad.

Encontramos varios efectos que resultaron significativos, entre ellos, la triple interacción entre tabaco, país y tipo 1 (Lambda de Wilks, $\left.F_{(2,149)}=4,2, p<0,05, \eta_{p}^{2}=0,053\right)$.

De la misma manera, aparece un efecto principal para el país (Lambda de Wilks, $F_{(2,149)}=$ $\left.15,2, p<0,001, \eta_{p}^{2}=0,170\right)$, y una interacción entre país y consumo de tabaco (Lambda de Wilks, $\left.F_{(2,149)}=5,1, p<0,01, \eta_{p}^{2}=0,064\right)$. Las pruebas de los efectos inter-sujetos (ANOVAs univariados) ponen de manifiesto que las diferencias se dan tanto para la primera medida de cortisol (i.e., cortisol-1) como para la segunda (i.e., cortiso1-2).

La Figura 1 y la Tabla 2 muestran las medias en cortisol de los diferentes grupos considerados. En cuanto a la triple interacción, el análisis de los datos pone de manifiesto que las diferencias más importantes se dan entre los 9 estudiantes mexicanos fumadores con altas puntuaciones en el tipo 1 cuya media de cortisol es significativamente más alta que en cualquier otro caso $(47,92)$.

De manera más específica, en el caso de la muestra mexicana, aparece una interacción entre fumar y personalidad tipo $1\left(F_{(1,83)}=6,5, p\right.$ $\left.<0,05, \eta_{p}^{2}=0,073\right)$, de tal manera que aquellos estudiantes que fuman y tienen altas puntuaciones en personalidad son los que más cortisol muestran en saliva $(47,92)$. En el caso de los españoles, no hay ningún efecto que resulte significativo.

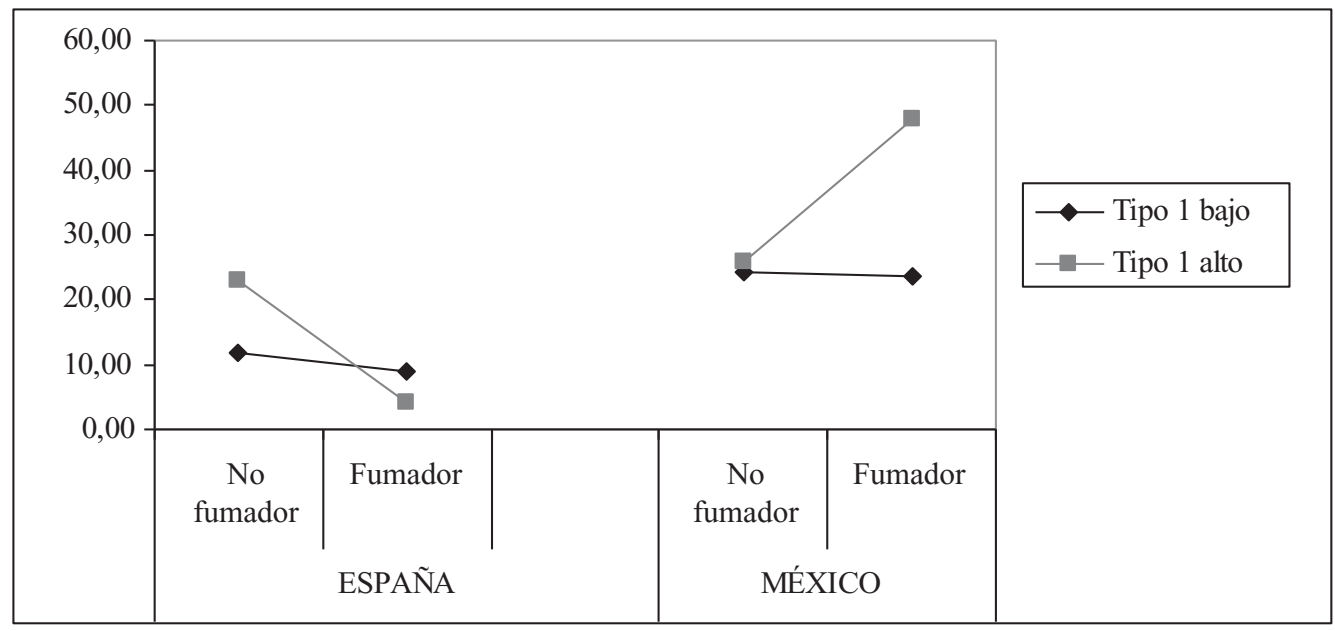

Figura 1: Niveles medios de cortisol-1 (nmol/L) según país, consumo de tabaco y tipo de personalidad

Tabla 2. Diferencia de medias en cortisol-1 (nmol/L) según país, consumo de tabaco y personalidad

\begin{tabular}{|c|c|c|c|c|c|c|c|c|}
\hline & \multicolumn{4}{|c|}{ España } & \multicolumn{4}{|c|}{ México } \\
\hline & \multicolumn{2}{|c|}{ No fumador } & \multicolumn{2}{|c|}{ Fumador } & \multicolumn{2}{|c|}{ No fumador } & \multicolumn{2}{|c|}{ Fumador } \\
\hline & Media & $D T$ & Media & $D T$ & Media & $D T$ & Media & $D T$ \\
\hline Tipo 1 bajo & 11,69 & 10,46 & 9,00 & 9,68 & 24,20 & 14,81 & 23,55 & 14,19 \\
\hline Tipo 1 alto & 22,94 & 19,63 & 4,15 & 8,68 & 25,74 & 18,67 & 47,92 & 28,55 \\
\hline
\end{tabular}


La interacción entre país y consumo de tabaco pone de manifiesto que, en el caso de los estudiantes mexicanos, aquellos que fuman tienen tendencia a presentar mayores niveles de cortisol (28,53 vs. 24,52). En el caso de los estudiantes españoles, la tendencia se invierte $\mathrm{y}$ los no fumadores suelen presentar mayores niveles de cortisol que los fumadores (13,41 vs. $8,70)$. Sin embargo, hay que destacar que en los contrastes intersujetos estas diferencias entre los grupos dentro de cada país desaparecen, de tal manera que el efecto más importante es el que pone de manifiesto las diferencias entre países.

Tal como hemos comentado, el principal efecto que se encuentra en esta investigación es la que hace referencia a las diferencias entre ambos países, de tal manera que en su conjunto, los estudiantes mexicanos muestran mayores niveles de cortisol en las dos medidas (Tabla 1).

Finalmente, mediante el segundo tipo de MANOVA llevamos a cabo análisis similares a los descritos arriba, si bien ahora incluyendo a la personalidad tipo 2 como variable independiente en el diseño (en lugar de la personalidad tipo 1). Estos análisis replicaron los resultados ya encontrados con los anteriores análisis sobre las diferencias entre países y la interacción entre país y consumo de tabaco. Sin embargo, no obtuvimos ningún resultado significativo asociado específicamente a la personalidad tipo 2 .

\section{DISCUSIÓN}

Durante la última década del siglo pasado se produjo cierta polémica sobre el papel de la personalidad en la predicción de la enfermedad, con numerosas aportaciones relacionadas con los tipos de personalidad descritos por Grossarth-Maticek y Eysenk (Orejudo y Froján, 2004; Sandín, 1995; Sebastián, León y Hospital, 2009). El propio Eysenck (1991c) llegó a proponer el papel mediador del cortisol entre el afrontamiento a los estresores por parte de las personas que presentaban personalidades de riesgo y la aparición de la patología. En este trabajo hemos tratado de poner parcialmente a prueba esta hipótesis, comparando los niveles de cortisol de estudiantes españoles y mexica- nos en función de sus características de personalidad según el modelo de Grossarth-Maticek y Eysenck. La relación se potenciaría cuando convergiesen otros factores de riesgo, como por ejemplo el consumo de tabaco (Eysenck, 1991a). Nuestros resultados muestran una ligera relación general entre los niveles de cortisol y las puntuaciones en personalidad tipo 1 , mientras que el efecto de interacción entre el tabaco y la personalidad aparece únicamente en el caso de los estudiantes mexicanos. No obstante, el efecto más importante que se encuentra es el relativo al país. Los estudiantes mexicanos muestran globalmente y de manera significativa mayores niveles de cortisol que los españoles (casi una desviación típica por encima). Por lo que respecta al tipo 2 de propensión cardiovascular, no se encuentran asociaciones directas con el cortisol ni el efecto de interacción con fumar. Este dato podría avalar la idea de que aún cuando apenas existen diferencias en los síntomas y las conductas de salud relacionadas entre el tipo de predisposición al cáncer y el cardiovascular (Orejudo y Froján, 2005; Orejudo et al, 2007), lo que hace que en ocasiones no se puedan diferenciar entre sí (Amelang y Schmidt-Rathjens, 1992), los datos de este trabajo podrían avalar las hipótesis iniciales de una distinta predisposición a la enfermedad de ambos tipos (Eysenck, 1991c; Sandín, 1995). Esto es, ambos tendrían características comunes y diferenciales que explicarían su común denominador psicopatológico, pero igualmente elementos diferenciales que podrían explicar una distinta predisposición.

Un primer análisis de estos datos nos llevaría a minimizar el papel de los factores de personalidad en el cortisol. Sin embargo, un análisis más detallado nos lleva a matizar esta visión inicial. Así, en general, los trabajos de Grossarth-Maticek y Eysenck han puesto de manifiesto que la personalidad resulta mejor predictor cuando nos encontramos con muestras estresadas (Eysenck, 1991,a,c; Grossarth-Maticek et al., 1988). Además, como hemos puesto de manifiesto en la introducción, los estudios transversales con muestras de personas adultas con estudios de casos y controles o similares, muestran más apoyo a la tipología de Eysenck y Grossarth-Maticek (Nagano et al., 2004; 
Matthews et al, 2003; Quander-Blaznik, 1991; Schmitz, 1992) que aquellos que se hacen con poblaciones sanas (Hirokawa et al., 2004; Sebastián y Miret, 2009; Terada et al., 2000). Una explicación plausible podría ser que se encuentran diferencias entre ambos países, y que la personalidad y el consumo de tabaco actúan como potenciados del estrés entre aquellas personas más estresadas, que por lo tanto presentarían mayores niveles de cortisol.

En aras de entender la importancia que la personalidad puede tener en el afrontamiento de los estresores hemos de señalar que el mismo Eysenck señala que los tipos de personalidad se definen a partir de las reacciones generadas ante los estresores (Eysenck, 1991a; Grossarth-Maticek et al, 1988); el trabajo de Orejudo et al. (2004) pone de manifiesto como éstos difieren notablemente en aspectos ligados al manejo del estrés, tales como los estilos de afrontamiento o el apoyo social. En este sentido, en nuestro trabajo aparecen algunos datos sobre la importancia del afrontamiento de la propia tarea como queda de manifiesto al comprobar cómo entre la primera y la segunda medida se produce una notable reducción de los niveles de cortisol en el caso de los estudiantes mexicanos. Es frecuente considerar los exámenes como estresores entre los estudiantes (Guerrero y Palmero, 2006), así como las exposiciones en público (García de la Banda et al., 2004). En este sentido, es posible que los estudiantes de nuestra investigación reaccionasen ante la novedad que podría suponer para ellos la toma de las muestras de saliva y la partición en la investigación, pero en cualquier caso este estresor sería de una magnitud relativamente reducida, ya que, difícilmente sería equiparable a la realización de un examen. De la misma manera, los resultados de trabajos como éste podrían ser de mayor magnitud si se realizasen bajo situaciones de estrés, tal como se propone desde la tipología de personalidad.

Como ya hemos comentado, otro resultado que ha aparecido es el relativo a la interacción entre el consumo de tabaco y el país. Recordemos que la nicotina introducida al organismo promueve el incremento en los niveles de cortisol per se (Kudielka, et al., 2009). Sin embargo una posibilidad que pudiera explicar los datos es que en términos culturales el acto de fumar puede tener una funcionalidad diferente. Es decir, en el contexto de los sujetos españoles el consumo de tabaco está asociado a la conversación y al momento de compartir un momento de relajamiento. En el contexto mexicano, el fumar puede ser un comportamiento generado por la ansiedad. Aportaciones generadas desde la neuromercadología (Lindstrom, 2008) identifican que cuando los fumadores hacen contacto visual con el letrero en la cajetilla que señala que el producto puede ser dañino para su salud se activa la zona de la amígdala en el cerebro del consumidor, correlato inequívoco de ansiedad. Cuando el fumador está ansioso fuma y por lo tanto el letrero referido se vuelve estratégico en la promoción de las ventas. Tanto la hipótesis de la funcionalidad cultural del fumar como el hecho de que los niveles de cortisol son altos solamente en fumadores con personalidad tipo 1 de un subgrupo, quedan por comprobarse con evidencia empírica.

En cualquier caso, queda patente que las personas presentan notables diferencias en sus respuestas de cortisol y que éste puede ser un importante factor de riesgo para la enfermedad. Hoy en día existe considerable evidencia que sugiere que el estrés tiene efectos importantes en la patofisiología de la enfermedad (Chrousos y Gold, 1998; McEwen, 1998; McEwen y Stellar, 1993), por ejemplo, véase el número especial del Journal of Consulting and Clinical Psychology al respecto, en donde se aborda el conocimiento psicológico respecto de diversas enfermedades (Smith, Kendall y Keefe, 2002). Análisis enfocados a enfermedades particulares como enfermedad cardíaca, diabetes y artritis reumatoide son desarrollados por Liegey y Baum (2001). Se asocian con el estrés agudo, las úlceras gástricas y los estados postquirúrgicos de recuperación (Camacho, Espinoza y Vega-Michel, 2012; Liang, Gao y Yin, 2008) y cicatrización lenta y con el estrés crónico el insomnio, colitis, migraña, hipertensión arterial, depresión y trastornos sexuales solamente por mencionar algunas de las enfermedades claramente identificadas. Se reconoce que la sobreactivación del eje hipotálamo-hipófisocorticosuprarrenal (HHC), genera inhibición del sistema inmune en el torrente sanguíneo, 
haciendo al organismo más vulnerable a infecciones virales y bacterianas (Straub, Buttgereit y Cutolo, 2011; Pace y Heim, 2001; Aguilera, 2001; Herbert y Cohen, 1993; Schleifer y Ke1ler, 1992; Irwin, 1992; Kennedy, Kiecolt-Glaser y Glaser, 1988). Esto se debe en buena parte al exceso de cortisol liberado por las glándulas adrenales como el último eslabón de la cadena de respuestas del eje HPA y que modifica la composición bioquímica a nivel humoral.

Siendo esto así, sigue siendo imprescindible investigar y conocer los factores que pueden explicar las diferencias en las respuestas de cortisol. Distintos trabajos insisten en la importancia de las diferencias individuales (García de la Banda et al., 2004), pero también en función de distintos estilos de vida. Se considera que la determinación de respuestas altas de estrés está modulada por las condiciones estresantes particulares, las condiciones de competencia de los sujetos y las variables de personalidad. Al respecto, Molloy, Perkins-Porras, Strike y Steptoe (2008) plantean que las variables de personalidad determinan el $6 \%$ de la varianza del cortisol y de ahí habría que poner en este contexto los hallazgos sobre las características de personalidad, como ocurre por ejemplo con el neuroticismo o la extraversión, factores que claramente ponen de manifiesto su efecto en contextos de estrés como se ha enfatizado en la introducción. En síntesis, el estrés y el afrontamiento del mismo, unido a diferentes estilos de vida, podrían ser factores activadores de la respuesta fisiológica de estrés. En este sentido, una línea de investigación que nos ayudase a avanzar sería poder identificar aquellas variables relacionadas con los estilos de vida o con factores culturales que pudiesen explicar las diferencias encontradas entre ambos países, el resultado más relevante encontrado en nuestra investigación.

Por último, queremos señalar algunas limitaciones de este trabajo. Así, por un lado, las muestras no se han obtenido por procedimientos aleatorios ni son directamente comporables en cuanto al tipo de titulación, aunque en ambos casos se trata de estudiantes universitarios con características similares en cuanto a edad y tipos de personalidad, pero no así en cuanto a su estilo de vida. Por otra parte, el análisis de las muestras de cortisol se han hecho en un mismo laboratorio, lo que en el caso de las muestras españolas conllevó un traslado, y aún cuando se mantuvieron buenas condiciones para el mantenimiento de las mismas, habrían podido sufrir alguna alteración. En cualquier caso, distintos trabajos ponen de manifiesto que el cortisol puede permanecer estable incluso a temperatura ambiente durante días (Donzella, Talge, Smith y Gunnar, 2008; Hansen et al., 2008). Por otra parte, el distinto método de respuesta de las respuestas de los cuestionarios de personalidad, en formato papel para los españoles y electrónico para los mexicanos, podría ser otra variable extraña que hubiese podido incidir en los resultados.

\section{REFERENCIAS}

Aguilera, G. (2011). HPA axis responsiveness to stress: implications for healthy aging. Experimental Geronto$\log y, 46,90-95$.

Alvaro, T. y Traver, T. (2010). Una revisión psiconeuroinmunológica de la fribromialgia. Revista de Psicopatología y Psicología Clínica, 15, 149-163.

Amelang, M. (1991). Tales from Crvenka and Heidelberg. What about the Empirical Basis. Psychological Inquiry, 2, 233-236.

Amelang, M. \& Schmitdt-Rathjens, C. (1992). Personality, Stress and Disease. Some Results on the Psychometric Properties of the Grossarth-Maticek and Eysenck Inventories. Psychological Reports, 71, 1251-1263.

Amelang, M. \& Schmitdt-Rathjens, C. (1996). Personality, cancer and coronary hearth disease. Further evidence on a controversial issue. British Journal of Health Psychology, 1, 191-205.

Amelang, M. (1997). Using personalty variables to predict cancer and heart disease. European Journal of Personality, 11, 319-342.

Camacho, E., Espinosa, A., y Vega Michel, C. (2012). Efectos de la imaginación dirigida y suministro de información en ansiedad y cortisol de pacientes ortopédicos. Revista Enseñanza e Investigación en Psicología, 17, 405-414.

Chorusos, G.P. \& Gold, P.W. (1998). A Helthy body in a helaty mind-and viceversa- the damaging power of «uncontrollable» stress. Journal of Clinical Endocrinology and Metabolism, 83, 1842-45.

Consoli, S.M., Cordier, S. \& Ducimetiére, P. (1993). Validation d'un Questionnaire de Personalité Destiné à Repérer des Sous-Groupes à Risque de Cardiopathie 
Ischémique ou de Cancer dans la Cohorte Gazel. Revue de Epidemiologie et de Sante Publique , 41, 315-326.

Donzella, B., Talge, N., Smith, T., \& Gunnar, M. (2008). To Spear or not to spear: comparison of saliva collection methods. Developmental Psychobiology, 50, 714717

Eysenck, H.J. (1987a): Personality, stress and cancer: Predictionand prophylaxis. Special issue: Stress and Health. British Journal of Medical Psychology, 61, 57-75.

Eysenck, H.J. (1987b): Personality as a predictor of cancer and cardiovaslcular disease, and aplicaction of behavior therapy in prophylaxis. European Journal of Psychiatry, 1, 29-41.

Eysenck, H.J. (1988). The respective importance of personality, cigarette smoking and interaction effects for the genesis of cancer and coronary hearth disease. Personality and Individual Differences, 9, 453-464.

Eysenck, H.J. (1991a). Smoking, Personality and Stress. Springer-Verlag. New York.

Eysenck,H.J. (1991b). Personality, stress and disease. An interactionist perspective. Psycohological Inquiry, 2, 221- 232

Eysenck H.J. (1991c). Cancer and personality. En C.L. Cooper y M. Watson (Eds.) Psycohological, Biological and Copyng Studies (pp.73-94). John Viley and Sons.

Eysenck, H.J. (1991d). Analysis of mortality data in the 1972 prospective Heilderberg study by GrossarthMaticek covering the period 1982-1986. Psycohological Inquiry, 2, 320-321.

Eysenck, H.J. (1993). Prediction of cancer and coronary heart disease mortality by means of a personality inventory. Results of a 15-year follow-up study. Psychological Reports, 72, 499-516.

Eysenck, H.J. \& Grossarth-Maticek, R. (1991). Creative Novation Behaviour Therapy as a prophylactic treatment for cancer and coronary heart disease. Part II. Effects of treatment. Behavior Research and Therapy, 29, 1 17-31.

Eysenck, H.J., Grossarth-Maticek, R. \& Everitt, B. (1991). Personality, stress, smoking, and genetic predisposition as synergistic risk factors for cancer and coronary heart disease. Integrative Physiological and Behavioral Science, 26, 4 309-322.

Fernández del Río, E., López, A. y Becoña, E. (2011). Resumen de Trastornos de personalidad en fumadores y personas con dependencia de la cocaína que acuden a tratamiento: un estudio comparativo. Revista de psicopatología y psicología clínica, 16, 67-75.

Flebus, G.B. y Cherri, G. (2003). Versione Italiana del Personality Stress Inventory di Grossarth-Maticek e Eysenck.Giornale Italiano di Psicologia, 30, 849-874.

García de la Banda, G., Martínez-Abascal, M.A., Pastor, M., Pérez y Doctor, R. (2004). Extraversion and neuroticism as predictors of salivary cortisol levels in public speaking. Análisis y Modificación de Conducta, 134, 935-948.

Grossarht-Maticek, R. \& Eysenck, H.J. (1995). Self-regulation and mortality from cancer, coronary heart diseae, and other cause. A prospective study. Personality and Individual Differences, 16, 781-795.

Grossarth-Maticek, R. \& Eysenck, H.J. (1991). Creative Novation Behaviour Therapy as a prophylactic treatment for cancer and coronary heart disease. Part I. Description of Treatment. Behavior Research and Therapy, 29, 1 1-16.

Grossarth-Maticek, R., Eysenck, H.J. Boyle, G.J., Heeb, J., Costa, S.D. \& Diel, J. (2000). Interaction of psychosocial and physical risk factors in the causation of mammary cancer, and its prevention through psychological methods of treatment. Journal of Clinical Psychology, 56, 33-50.

Grossarth-Maticek, R., Eysenck, H.J. \& Vetter, H. (1988). Personality type, smoking habit and their interaction as predictors of cancer and coronary hearth disease. Personality and Individual Differences, 9, 479-495.

Guerrero, C. y Palmero, F. (2006). Percepción de control y respuestas cardiovasculares. International Journal of Clinical and Health Psychology, 6,145-168.

Hansen, A., Garde, A. \& Persson, R. (2008). Sources of biological and methodological variation in salivary cortisol and their impact on measurement among healthy adults: a review. Scandinavian Journal of Clinical \& Laboratory Investigation, 68, 448-458.

Hart, D., Burock, D., London, B., Atkins, R. \& BonillaSantiago, G. (2005). The relation of personality types to physiological, behavioural and cognitive processes. European Journal of Personality, 19, 391-407.

Hauner, K., Adam, E., Mineka, S., Doane, L., DeSantis, A., Zinbarg, R., Craske, M. \& Griffith, J. (2008), Neuroticism and introversión are associated with salivary cortisol patterns in adolescents. Psychoneuroendochrinology, 33, 1344-1356.

Herbert, T. \& Cohen, S. (1993). Depression and immunity: A meta-analytic review. Psychological Bulletin, 11, 472-486.

Herbert, T. \& Cohen, S. (1993). Depression and immunity: A meta-analytic review. Psychological Bulletin, 11, 472-486.

Hirokawa, K., Nagata, C., Takatsuka, N. \& Shimizu, H. (2004). The relationship of a rationality/antiemotionality personality scale to mortalities of cancer and cardivascular disease in a community population in Japan. Journal of Psychosomatic Research, 56, 103111.

Irwin, M. R. (1992). Depression: Role of Corticotropin Releasing Factor in the reduction of natural killer cell activity. En H. Schmoll, U. Tewes, \& N. Plotnikoff (Eds.). Psychoneuroimmunology: Interactions between Brain, Nervous System, Behavior, Endocrine and Im- 
mune System. (pp. 107-126). New York: Hogrefe \& Huber Publishers.

Kennedy, S., Kiecolt-Glaser, J., \& Glaser, R. (1988). Immunological consequences of acute and chronic stressors: Mediating role of interpersonal relationships. British Journal of Medical Psychology, 61, 77-85.

Kiecolt-Glaser, J.K. \& Chee, M.A. (1991). Personality, Stress and Cancer. A Re-examination. Psycohological Inquiry, 2, 249-251.

Kiecolt-Glaser, J.K., McGuire, L., Robles, T.F. \& Glaser, R. (2002). Psychoneuroimmunology and psychosomatic medicine: Back to the future. Psychosomatic Medicine, 64, 15-28.

Kirk, K.M., \& Martín, N.G. (1998). The short Interpersonal Reactions Inventory, Self-regulation and Differentiation scales in an older Australian twin sample. Personality and Indidual Differences, 25, 591-604.

Kirschbaum, C., \& Hellhammer, D. (1989). Salivary cortisol in Psychobiological research. an overview. Neuropsychobiology, 22, 150-169.

Kudielka, B.M., Federanko, I.S., Hellhammer, D.H. \& Wüst, S. (2006). Morningness and eveningness. The free cortisol rise after awakening in «early birds» and «night owls». Biological Psychology, 72, 141-146.

Kudielka, B., Hellhammer, D. \& Wüst, S. (2009). Why do we respond so differently? Reviewing determinants of human salivary cortisol responses to challenge. Psychoneuroendochrinology, 34, 2-18.

Larsson, G., Nordström, L, Ljunggren, G. \& Nyberg, A.B. (1995). The Grossarth-Maticek and Eysenck personality types, health-related behaviour, and indicators of transitory ill-health. European Journal of Personality, 9, 75-87.

Liang, P., Gao, X. \& Yin, J. (2008). Assessment of preoperative psychological state of patients with liver transplantation. Zhonghua Yi Xue Za Zhi, 88, 3045-3048.

Liegey, A. \& Baum, A. (2001). Stress, Health and Illness. In A. Baum, T. Revenson Singer (Eds.) Handbook of Health Psychology. New Jersey, U.S.A. : Lawrence, Erlbaum Associates Publishers.

Lindstrom, M. (2008). Buyology: Truth and Lies about Why we buy. New York, U.S.A.: Doubleday.

Loney, B., Butler, M., Lima, E., Counts, C. \& Eckel, L. (2006). The relation between salivary cortisol, callousunemotional traits, and conduct problems in an adolescent non-referred simple. Journal of Child Psychology and Psychiatry, 47, 30-36.

Martínez, A. y Reyes, G.A. (2004). Personalidad y factores clásicos de riesgo en la predicción de la enfermedad somática. un análisis de la validez convergente y discriminante del modelo de Eysenck y Grossarth-Maticek en sujetos análogos. Análisis y Modificación de Conducta, 30, 349-403.

Martínez, A. y Reyes, G.A. (2007). Reliability, factorial validity and normative data of the Spanish Short Inter- personal Reactions Inventory (SIRI) by GrossarthMaticek and Eysenck. Revista de Psicopatología y Psicología Clínica, 12, 131-142.

Matthews, G., Yousfi, S. Schmidt-Rathjens, C. \& Amelang, M. (2003). Personality Variable Differences Between Disease Clusters. European Journal of Personality, Journal of Personality, 17, 157-177.

McEwen, B.S. \& Stellar, E. (1993). Stress and the individual. Mechanisms leading to disease. Archives of Internal Medicine, 153, 2093-2101.

McEwen, B. (1998). Protective and damaging effects of stress mediators. The New England Journal of Medicine, 338, 171-181.

Molly, G., Perkins-Porras, L., Strike, P. \& Steptoe, A. (2008). Type D personality and cortisol in survivors of acute coronary syndrome. Psychosomatic Medicine, 70, 863-868.

Nagano, J., Nagase, S., Sudo, N. \& Kubo, C. (2004). Psychosocial Stress, Personality, and the Severity of Chronic Hepatitis C. Psychosomatics. Journal of Consultation Liaison Psychiatry, 45, 100-106.

Orejudo, S., Froján, M. X. y Malo, C. (2004). La tipología de personalidad de Grossarth-Maticek y Eysenck. Relación con otros constructos psicológicos. Psiquis, 25,152-166.

Orejudo, S. y Froján, M.X. (2005). Síntomas somáticos. Predicción diferencial a través de variables psicológicas, sociodemográficas, estilos de vida y enfermedades. Anales de Psicología, 21, 276,285.

Orejudo, S., Froján, M.X. y Malo, M.C. (2007). Illnes behavior. Prediction by simptoms complaints, the Grossarth-Maticek and Eysenck personality types, neuroticism, life events, coping, health locus of control, social support and atributional style. Spanish Journal of Psychology, 10, 388-398.

Oswald, L., Zandi, P., Nestadt, G., Potash, J., Kalaydjian, A. \& Wand, G. (2006). Relationship between cortisol responses to stress and personality. Neuropsychopharmacology, 31, 1583-1591.

Pace, T., \& Heim, C. (2001). A short review on the psychoneuroimmunology of posttraumatic stress disorder: from risk factors to medical comorbidities. Brain, Behavior and Immunity, 25, 6-13.

Pelossi, A.J. y Appleby, L. (1992). Psychological Influences on Cancer and Ischaemic Disease. British Medical Journal, 304, 1295-1298.

Portella, M., Harmer, C., Flint, J., Cowen, P. \& Goodwin, G. (2005). Enhanced early morning salivary cortisol in neuroticism. American Journal of Psychiatry, 162, 807-809.

Pruessner, J., Gaab, J., Hellhammer, D., Lintz, D., Schommer, N. \& Kirschbaum, C. (1997). Increasing correlations between personality traits and cortisol stress responses obtained by data aggregation. Psychoneuroendochrinology, 22, 615-625. 
Quander-Blaznik, J. (1991). Personality as Predictor of Lung Cancer. A Replication. Personality and Individual Differences, 12, 125-130.

Ranchor, A. \& Sanderman, R. (1991). The role of personality and socio-economic status in the stress-illness relation. A longitudinal study. European Journal of Personality, 5, 93-108.

Rodríguez, A.M., Lemos, S. y Canga, A. (2001). Relación entre variables de personalidad, actitudes hacia la salud y estilo de vida. Revista de Psicología General y Aplicada, 54, 659-670.

Rodríguez, A.M., Lemos, S. y Canga, A. (2002). Variables biológicas, conductuales y de personalidad como predictores de salud. Un estudio longitudinal. Psicología Conductual, 10, 7-29.

Sandín, B. (1995). El estrés. En A. Belloch, B.Sandín y F. Ramos (Eds.). Manual de Psicopatología, Vol. 2 (pp. 3-52). Madrid: McGraw-Hill

Sandín, B., Chorot, P., Navas, M.J. y Santed, M.A. (1992). Estrés y Enfermedad. Inventario de Reacciones Interpersonales de Grossarth-Maticek y Eysenck. Revista de Psicología General y Aplicada, 45, 391-396.

Sandín, B., Chorot, P., Santed, M.A. y Jiménez, M.P. (1994). Stress Behavior Types, Personality, Alexthymia, Copyng and State-Trait Anger Expresion. Stress News, 5 (4), 2-8.

Sandín, B., Chorot, P., Jiménez, M.P. y Santed, M.A. (1994). Stress Behavior Types, Psychosomatic Complaints and Disease. Stress News, 5 (3), 4-9.

Schleifer, S. J., \& Keller, S. E. (1992). Stressful events, Depressive Disorders, and Immunity. En H. Schmoll, U. Tewes, y N. Plotnikoff (Eds.). Psychoneuroimmunology: Interactions between Brain, Nervous System, Behavior, Endocrine and Immune System. (pp. 91-99). New York: Hogrefe \& Huber Publishers.

Schmitz, P.G. (1992). Personality, Stress-Reactions and Disease. Personality and Individual Differences, 13, 683-691.
Sebastián, J., León, M. y Hospital, A. (2009).Variables psicosociales y cáncer de mama. Un estudio cuasiprospectivo de la personalidad tipo C. Revista Latinoamericana de Psicología, 41, 461-479.

Sebastián, J. y Miret, M. (2009). De los trabajos de Grossarth-Matick y Eysenck a las escalas de Spilbergerger. Su aplicación en mujeres con cáncer de mama. Psicología Conductual, 17, 453-479.

Smedslund, G. (1995). Personality and vulneravility to cancer and heart disease. Relations to demographic and life-style variables. Personality and Individual Differences, 19, 691-697.

Smith, T., Kendall, P., \& Keefe, F. (2002). Special Issue: Behavioral Medicine and Clinical Health Psychology. Journal of Consulting and Clinical Psychology, 70 (3), 459-856. Doi: 10.1037//0022-006X.70.3.459

Straub, R., Buttgereit, F., \& Cutolo, M. (2011). Alterations of the hypothalamic-pituitary-adrenal axis in systemic immune diseases - a role for misguided energy regulation. Clinical and Experimental Rheumatology 5, 23-31.

Terada, K., Kawakami, N., Inaba, S., Takatsuka, N. \& Shimizu, H. (2000). Rationality/antiemotionality personality and selected chronic diseases in a community population in Japan, Journal of Psychosomatic Research, 48, 31-35.

Vermunt, R., Peeters, Y. \& Berggren, K. (2007). How fair treatment affects saliva cortisol release in stressed low and high type A behavior individuals. Scandinavian Journal of Psychology, 48, 547-555.

Zobel, A., Barkow, K., Schulze-Rauschenbach, S., von Widdern, O., Metten, M., Pfeiffer, U. et al. (2004). High neuroticism and depressive temperament are associated with dysfunctional regulation of the hypothalamic-pituitary-adrenocortical system in healthy volunteers. Acta Psychiatrica Scandinavica, 109, 392-399. 